\title{
In situ and real time determination of metallic and semiconducting single-walled carbon nanotubes in suspension via dielectrophoresis
}

\author{
Natacha Mureau, Ernest Mendoza, and S. R. P. Silva \\ Advanced Technology Institute, Nano-Electronics Centre, University of Surrey, Guildford, \\ Surrey GU2 7XH, England \\ Kai F. Hoettges and Michael P. Hughes ${ }^{a)}$ \\ Centre for Biomedical Engineering, University of Surrey, Guildford, Surrey GU2 7XH, England
}

(Received 25 January 2006; accepted 12 April 2006; published online 13 June 2006)

\begin{abstract}
We report an alternative method combining dielectrophoresis and impedance spectroscopy to provide rapid, accurate measurement of dielectrophoretic collection of single-walled carbon nanotubes (SWNTs) in real time. We analyzed a Triton X-100 suspension of mixed SWNTs to measure their precise dielectrophoretic collection behavior. Results indicate that our sample contains $21.5 \%$ of metallic and $78.5 \%$ of semiconducting carbon nanotubes before separation, and that the range of $1-15 \mathrm{MHz}$ is ideal to collect only the metallic ones. Optical absorption was used to confirm these proportions. We discuss the possible errors associated with using purely a Raman technique to validate the type of SWNTs collected in suspension. (c) 2006 American Institute of Physics. [DOI: 10.1063/1.2207501]
\end{abstract}

Since their discovery by Iijima, ${ }^{1}$ carbon nanotubes have demonstrated great potential in nanoelectronic applications due to their remarkable chemical, physical, and electrical properties. ${ }^{2}$ Depending on their diameter and chirality, single-walled carbon nanotubes (SWNTs) can behave either as a metal or a semiconductor. Dielectrophoresis ${ }^{3}$ (DEP), the movement of particles in nonuniform electric fields, has been used to separate mixtures of semiconducting and metallic SWNTs. ${ }^{4-6}$ Measurement of the frequency-dependent collection of DEP has thus far been based on Raman spectroscopy, which requires some complex interpretation of results. ${ }^{7-9}$ In this letter, we report the application of combined dielectrophoresis and impedance measurements to provide real time rapid, accurate measurement of dielectrophoretic collection of SWNTs, and hence their dielectric properties and chiral proportions.

SWNTs were made by laser ablation and suspended in a Triton X-100 solution. Measurements were performed on needle-shaped electrodes with a $10 \mu \mathrm{m}$ gap, energized with a sinusoidal $10 \mathrm{~V}_{\mathrm{pk}-\mathrm{pk}}$, at five or more frequencies per decade between $10 \mathrm{kHz}$ and $20 \mathrm{MHz}$. A resistance was connected in series, and the voltage measured across the resistor and resistor/electrode combination using a digital oscilloscope. The oscilloscope output to a computer, which calculated the resistance of the interelectrode gap at $1 \mathrm{~s}$ intervals. The recording process began prior to the SWNTs suspension being placed on the electrodes, and continued for typically $5 \mathrm{~min}$. The time constant of the change of resistance as a function of time was then obtained.

Figure 1(a) shows a representative selection of impedance versus time data which has been normalized to the electrode impedance. The solution conductivity was measured at $1.3 \mathrm{mS} \mathrm{m}^{-1}$, causing a $30 \%$ decrease in resistance after application. Two types of behavior were observed in nanotube solutions. At high frequencies, an initial resistance drop at the application of the sample was followed by an exponential

\footnotetext{
a) Author to whom correspondence should be addressed; electronic mail:
} m.hughes@surrey.ac.uk decrease to a stable value at approximately $200 \mathrm{~s}$. The decrease in resistance (final with respect to initial values) was $53 \%$ for $1 \mathrm{MHz}, 39 \%$ for $2 \mathrm{MHz}$, and $48 \%$ for $5 \mathrm{MHz}$. At lower frequencies, the reduction was more significant, e.g., $88 \%$ for $20 \mathrm{kHz}, 79 \%$ for $50 \mathrm{kHz}$, and $67 \%$ for $100 \mathrm{kHz}$. At $20 \mathrm{MHz}$, the response indicated that the impedance change was due to the medium alone, that is, the result was identical to the control measurement and no collection occurred.

If we hypothesize that the time taken for the impedance to change due to the collection of nanotubes is inversely proportional to the force acting on those tubes, then the reciprocal of the time constant should indicate the magnitude of the force. A similar approach has been taken using fluorescence measurements of latex beads to determine their dielectric properties. ${ }^{10}$ Across a population of particles, this reciprocal also indicates the relative population of subpopulations of particles. By modeling the population using known models of dielectrophoretic behavior, we determined the dielectric properties of each population and their relative number. ${ }^{11}$

A plot of the reciprocal collection time constant is shown in Fig. 1(b). Two dielectric dispersions are evident, centered approximately at $250 \mathrm{kHz}$ and $13 \mathrm{MHz}$. This is consistent with behavior we would expect for a heterogeneous mix of metallic and semiconducting SWNTs. ${ }^{11}$ For a spherical particle of radius $r$ the DEP force is given by

$$
F_{\text {sphere }}=2 \pi r^{3} \varepsilon_{m} \operatorname{Re}\left(\frac{\varepsilon_{p}^{*}-\varepsilon_{m}^{*}}{\varepsilon_{p}^{*}+2 \varepsilon_{m}^{*}}\right) \nabla E^{2},
$$

where $\varepsilon_{m}$ is the permittivity of the suspending medium, $E$ is the local (rms) electric field, $\nabla$ is the differential vector operator, $\varepsilon_{p}^{*}$ and $\varepsilon_{m}^{*}$ are the complex permittivities of the particle and medium, respectively, $\varepsilon^{*}=\varepsilon-j(\sigma / \omega)$, where $\varepsilon$ is the permittivity, $\sigma$ is the conductivity, $\omega$ is the angular frequency of the applied field, $j=\sqrt{-1}$, and Re denotes the real part. For a prolate ellipsoid with major axis $r_{1}$ and minor axis $r_{2}$, the force is given by 

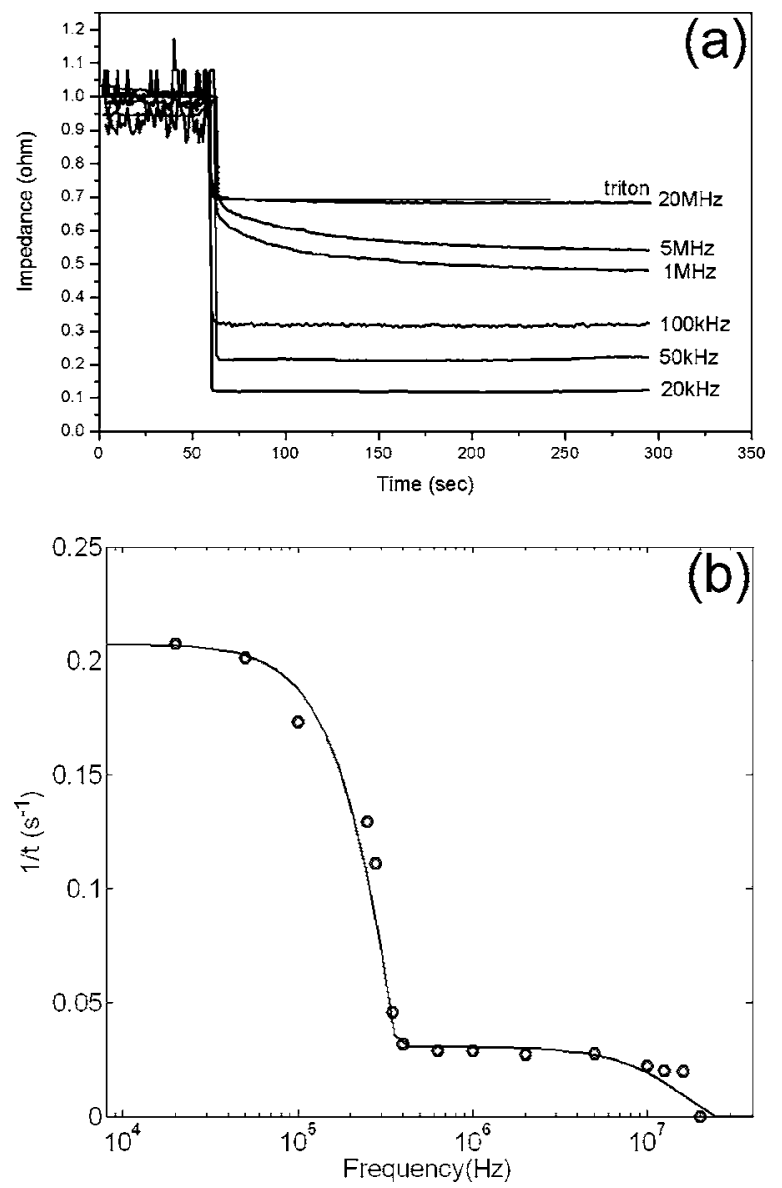

FIG. 1. Experimental results of impedance measurements. (a) Plot of impedance as a function of time, after normalization. Application of the nanotube suspension is clearly visible at $60 \mathrm{~s}$. (b) Plot of reciprocal collection time constant as a function of frequency (dots) with the predicted value (solid line) using a model of the dielectrophoretic collection.

$$
F_{\text {rod }}=\frac{2 \pi r_{1} r_{2}^{2} \varepsilon_{m}}{3} \operatorname{Re}\left(\frac{\varepsilon_{p}^{*}-\varepsilon_{m}^{*}}{\varepsilon_{m}^{*}}\right) \nabla E^{2}
$$

Based on the relative magnitudes of $\varepsilon_{p}^{*}$ and $\varepsilon_{m}^{*}$, which are in turn related to $\omega$, the DEP force acting on a particle can cause it to move either towards or away from high-field regions at electrode edges. These two effects are termed positive and negative DEP, respectively.

The solid line on Fig. 1(b) represents the best fit for two populations of nanotubes, superimposed to determine the net frequency-dependent SWNTs collection. A spherical model, postulated by Krupke et al. ${ }^{4}$ to account for ballistic ion transport, was found to provide a good fit for the conducting nanotubes, when the nanotubes were assigned a conductivity of $130 \mathrm{mS} \mathrm{m}{ }^{-1}$ and a relative permittivity of less than $40 \varepsilon_{0}$. We were able to obtain a unique set of parameters that enabled us to model semiconducting nanotubes either as spheres or as long, thin ellipsoids - an approach that has been used for dielectrophoretic modeling of rod-shaped nanoparticles in the past. ${ }^{12}$ Both models have been proposed for the description of the DEP behavior of SWNTs by Krupke et al. ${ }^{4,6}$

In the case of a spherical model, the semiconducting nanotubes had a conductivity of $2.2 \mathrm{mS} \mathrm{m}^{-1}$, and for the prolate ellipsoid model, $2.5 \mathrm{mS} \mathrm{m}^{-1}$. As with the conducting nanotubes, the model indicates a permittivity of less than $40 \varepsilon_{0}$. Notably, this permittivity is considerably lower than Downloaded 30 Mar 2009 to 131.227.178.132. Redistribution subje

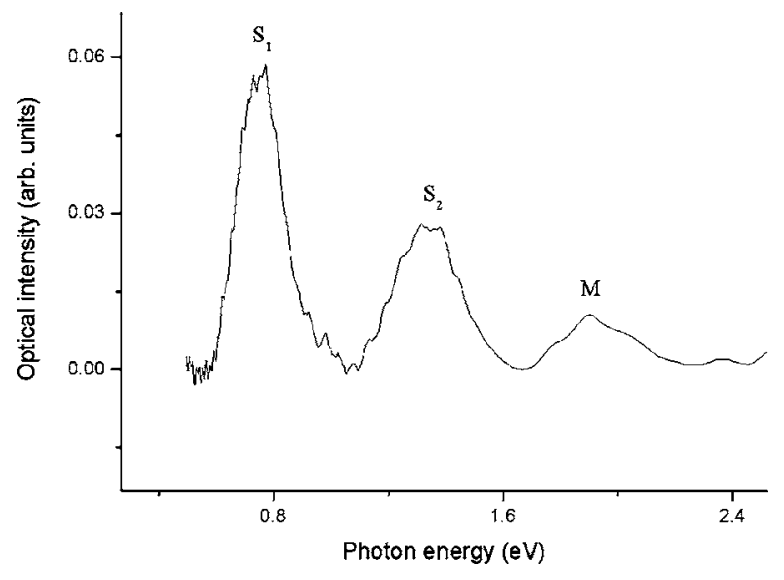

FIG. 2. Optical absorption spectrum of the SWNTs before dielectrophoresis. Background absorption due to the $\pi$ plasmon was corrected.

the near-infinite value suggested in the literature, ${ }^{13}$ though larger than that reported by Krupke et al. for micellated SWNTs. ${ }^{4,6}$ The values are more consistent when considered as an ensemble of nanotube plus its Debye atmosphere, which dominates the dielectric properties of nanoparticles in aqueous solutions. ${ }^{14}$ It is also known ${ }^{14}$ that the electrical double layer is a low-permittivity environment, the radius of which is of the order of the diameter of the SWNTs themselves. The presence of Triton on the SWNTs may also affect this value. Since the nanotubes vary only in chirality, we would expect both metallic and semiconducting SWNTs to have identical electrical Debye layer properties.

We can further determine the relative populations of the particles by determining the relative multiples of each required to match the spectra in Fig. 1(b). Our calculations indicate that where the semiconducting nanotubes are modeled as prolate ellipsoids, the proportions of the two polarizability plots in Fig. 1(b) indicate that the population contains $78.5( \pm 1 \%)$ semiconducting SWNTs, with the remainder being metallic. This proportion appears high at first, but is commensurate with the findings of Samsonidze et al. ${ }^{15}$ who showed that semiconducting nanotubes dissolved preferentially in Octadecylamine (ODA); if similar effects are present in Triton-dissolved nanotubes, we would anticipate the result described here. If the spherical model is used, the proportion of semiconducting nanotubes is much higher $(95 \pm 0.5 \%)$.

In order to validate these results, we used optical absorption, a theoretically valid technique for the determination of the ratio metallic:semiconducting SWNTs (Ref. 8) which has widely been employed for carbon nanotube characterization. ${ }^{16-19}$ UV/Vis/NIR spectroscopy was performed after drop casting the solution on a glass slide and heating to $200{ }^{\circ} \mathrm{C}$ for $1 \mathrm{~h}$. The spectrum is shown in Fig. 2, following base line correction. An average diameter of $1.3 \mathrm{~nm}$ was estimated from the first peak, corresponding to the $v_{1}-c_{1}$ singularity transition of semiconducting SWNTs (Ref. 18) [transmission electron microscopy (TEM) confirmed this value with a distribution ranging between 1.2 and $1.5 \mathrm{~nm}$ ]. The ratio between the first and the third peak (corresponding to the $v_{1}-c_{1}$ transition of metallic SWNTs) indicates a population of $79.6 \%( \pm 3 \%)$ of semiconducting SWNTs in solution before dielectrophoresis, which is in good agreement with our calculations when considering the semiconducting SWNTs as rodlike elements. This assumption is valid when we consider that the model of nanotubes to AIP license or copyright; see http://apl.aip.org/apl/copyright.jsp 


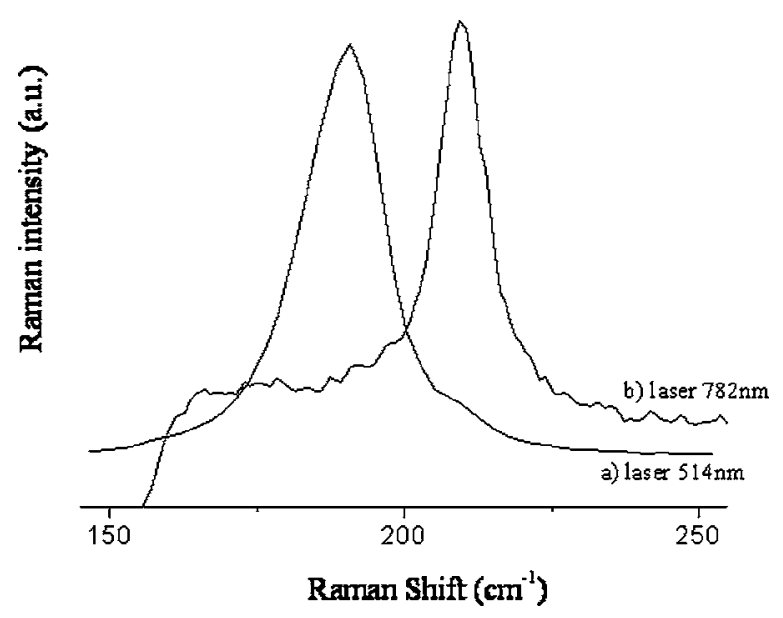

FIG. 3. Raman profiles of the SWNTs before dielectrophoresis. RBM regions recorded with (a) a $514 \mathrm{~nm}$ laser and (b) a $782 \mathrm{~nm}$ laser (averaging of ten spectra obtained in different spots on the sample).

as spheres only hold for ballistic transport, which does not take place in semiconducting SWNTs. This result also suggests that it is possible to determine the proportion of metallic and semiconducting SWNTs in a suspension using only two impedance measurements, at frequencies chosen so as to collect both types at low frequency, and only metallic nanotubes at high frequency. Such a method has significant potential to greatly simplify the methods available today for the analysis of SWNTs pre- and post-separation.

Raman spectroscopy was also used to characterize our samples and to calculate the ratio metallic:semiconducting SWNTs. We used a confocal Raman microscope with two lasers operating at 728 and $514 \mathrm{~nm}$. Krupke et al. ${ }^{4}$ previously concluded that, with a $514 \mathrm{~nm}$ laser and a wide nanotubes diameters distribution centred at $1.1 \mathrm{~nm}$, all metallic SWNTs have radial breathing mode (RBM) frequencies in the range of $218-280 \mathrm{~cm}^{-1}$ and all semiconducting SWNTs have RBM frequencies ranging between 175 and $213 \mathrm{~cm}^{-1}$. Then they applied a formula involving the ratio of the integrated peaks of metallic and semiconducting SWNTs to calculate their relative proportions. On the other hand, Kataura et $a{ }^{8}{ }^{8}$ predicted without ambiguity which types of SWNTs will be resonant with a specific laser excitation, depending on diameters and chirality. Based on the plots of Kataura et $a l$., we conclude that in order to get a quantitative measurement, Raman spectroscopy is useful only when the tubes have a broad diameter distribution centered at $1.1 \mathrm{~nm}$. However, where the diameter distribution is narrower and centered at higher value, it is not possible to excite both metallic and semiconducting tubes with the same laser energy; that is to say, the $514 \mathrm{~nm}$ laser will excite semiconducting tubes and conversely, the $782 \mathrm{~nm}$ laser will excite the metallic tubes. We confirmed this using measurements which are consistent with this theoretical prediction. With the $514 \mathrm{~nm}$ laser, we observe a peak in the RBM region below $200 \mathrm{~cm}^{-1}$ corresponding to semiconducting nanotubes (cf. Fig. 3, curve a). With the $782 \mathrm{~nm}$ laser, the spectrum shows a RBM band above $200 \mathrm{~cm}^{-1}$ attributed to metallic nanotubes (cf. Fig. 3, curve b). Therefore, with our distribution of diameters, neither of these two lasers will enable us to excite simultaneously the two types of SWNTs and thus to calculate their respective proportions. We suggest that under our experimental conditions, optical absorption is the only method that can be used to accurately validate our dielectrophoretic measurements. We believe this letter will highlight to the community the importance of understanding the methodology and limitations of each experimental technique used for the characterisation of SWNTs.

In conclusion, we have demonstrated a rapid, precise, and low-cost method for performing dielectric spectroscopy on SWNTs suspensions, allowing rapid, accurate determination of both the dielectric properties of the SWNTs and the proportions of metallic and semiconducting SWNTs. The precision afforded by the system has potential for providing more accurate control over defining the optimal conditions for the large-scale separation of SWNTs required for the adoption of nanotubes as a material of choice in the semiconductor industry.

This work was supported by EPSRC Grant No. GR/ S44594. The authors thank Ewa Borowiak-Palen for her help on optical absorption, Alan Dalton for supplying the SWNTs suspension, and Vlad Stolojan for the TEM analysis.

${ }^{1}$ S. Iijima, Nature (London) 354, 56 (1991).

${ }^{2}$ M. S. Dresselhaus, G. Dresselhaus, and Ph. Avouris, Carbon Nanotubes: Synthesis, Structure, Properties and Applications (Springer, New York, 2001).

${ }^{3}$ H. A. Pohl, Dielectrophoresis (Cambridge University Press, Cambridge, 1978).

${ }^{4}$ R. Krupke, F. Hennrich, H. von Löhneysen, and M. M. Kappes, Science 301, 344 (2003).

${ }^{5}$ D. S. Lee, D. W. Kim, H. S. Kim, S. W. Lee, S. H. Jhang, Y. M. Park, and E. E. B. Campbell, Appl. Phys. A: Mater. Sci. Process. 80, 5 (2005).

${ }^{6}$ R. Krupke, F. Hennrich, M. M. Kappes, and H. von Löhneysen, Nano Lett. 4, 1395 (2004).

${ }^{7}$ M. S. Strano, S. K. Doorn, E. H. Haroz, C. Kittrell, R. H. Hauge, and R. E. Smalley, Nano Lett. 3, 1091 (2003).

${ }^{8}$ H. Kataura, Y. Kumazawa, Y. Maniwa, I. Umezu, S. Suzuki, Y. Ohtsuka, and Y. Achiba, Synth. Met. 103, 2555 (1999).

${ }^{9}$ S. M. Bachilo, M. S. Strano, C. Kittrel, R. H. Hauge, R. E. Smalley, and R. B. Weisman, Science 298, 2361 (2002).

${ }^{10}$ D. J. Bakewell and H. Morgan, Meas. Sci. Technol. 15, 254 (2004).

${ }^{11}$ L. Broche, F. H. Labeed, and M. P. Hughes, Phys. Med. Biol. 50, 2267 (2005).

${ }^{12}$ H. Morgan and N. G. Green, J. Electrost. 42, 279 (1997).

${ }^{13}$ L. X. Benedict, S. G. Louie, and M. L. Cohen, Phys. Rev. B 52, 8541 (1995).

${ }^{14}$ M. P. Hughes, J. Colloid Interface Sci. 250, 291 (2002).

${ }^{15}$ Ge. G. Samsonidze, S. G. Chou, A. P. Santos, V. W. Brar, G. Dresselhaus, M. S. Dresselhaus, A. Selbst, A. K. Swan, M. S. Ünlü, B. B. Goldberg, D. Chattopadhyay, S. N. Kim, and F. Papadimitrakopoulos, Appl. Phys. Lett. 85, 1006 (2004).

${ }^{16}$ S. Maruyama, Y. Miyauchi, Y. Murakami, and S. Chiashi, New J. Phys. 5, 149.1 (2003).

${ }^{17}$ R. Krupke, F. Hennrich, H. B. Weber, M. M. Kappes, and H. von Löhneysen, Nano Lett. 3, 1019 (2003).

${ }^{18}$ X. Liu, T. Pichler, M. Knupfer, M. S. Golden, J. Kink, H. Kataura, and Y. Achiba, Phys. Rev. B 66, 045411 (2002).

${ }^{19}$ M. H. Rummeli, E. Borowiak-Palen, T. Gemming, T. Pichler, M. Knupfer, M. Kalbac, L. Dunsch, O. Jost, S. R. P. Silva, W. Pompe, and B. Buchner, Nano Lett. 5, 1209 (2005). 